\title{
The One-dimensional Problem of Unsteady-related Elastic Diffusion Layer
}

\begin{abstract}
A. R. Gachkevich ${ }^{1}$, A. V. Zemskov ${ }^{2}$, D. V. Tarlakovsky ${ }^{2}$
${ }^{1}$ Ya. S. Pidstryhach Institute for Applied Problems of Mechanics and Mathematics, Ukraine, 79060, L'vov, Naukova st., 3 b, dept13@iapmm.lviv.ua

${ }^{2}$ Moscow Aviation Institute (State University of Aerospace Technologies), Russia, 125993, Moscow, GSP-3, A-80, Volokolamskoe Shosse, 4, azemskov1975@mail.ru, tdvhome@mail.ru

The problem of determining the stress strain state of an elastic medium, taking into account the structural changes caused by the presence of diffusion fluxes. The influence of diffusion processes on the stress-strain state of the environment is taken into account by using the locally equilibrium model of thermoelastic diffusion, which includes the coupled system of equations of motion of an elastic body and the equations of heat and mass transfer. For solutions used decompositions of the unknown functions in Fourier series and then applying the integral Laplace transform with respect to time. We construct a fundamental solution of the problem. For examples the cases where the diffusion flux at the boundary is constant, or decays exponentially are considered.
\end{abstract}

Key words: elastic diffusion, time-dependent problems, Fourier series, Laplace transform.

\section{References}

1. Eremeev V. S. Diffuziia i napriazheniia [Diffusion and stresses]. Moscow, Moscow Univ. Press, 1978, 287 p. (in Russian).

2. Knjazeva A. G. Vvedenie v lokal'no-ravnovesnuiu termodinamiku fiziko-khimicheskikh prevrashchenii v deformiruemykh sredakh [Introduction to the locallyequilibrium thermodynamics of physical and chemical transformations in deformable environments]. Tomsk, 1996, 146 p. (in Russian).

3. Podstrigach Ja. S., Pavlina V. S. Differential equation of thermodynamic processes in the $n$-component solid solution. Fiziko-himicheskaja mehanika materialov, 1965, no. 4, pp. 383-389. (in Russian).
4. Bugaev N. M., Gachkevych A. R., Zemskov A. V., Tarlakovsky D. V. Approximated solution of one-dimensional bound thermo elastic diffusion problem for half space. Problemi obchisljuval'noi mehaniki $i$ micnosti konstrukcij: zbirnik naukovih prac', Dnipropetrovs'kij nacional'nij universitet, Dnipropetrovs'k, IMA-pres, 2011, iss. 16, p. 60-68. (in Russian).

5. Gorshkov A. G., Medvedsky A. L., Rabinsky L. N., Tarlakovsky D. V. Volny v sploshnykh sredakh Waves in Continuous Media. Moscow, Fizmatlit, 2004, 472 p. (in Russian).

УДК 51-73

\section{МЕТОДИКА ОПРЕДЕЛЕНИЯ ОБЛАСТЕЙ, ТРЕБУЮЩИХ КВАНТОВОГО ОПИСАНИЯ В РАМКАХ ГИБРИДНОГО МЕТОДА (КВАНТОВАЯ МЕХАНИКА / МОЛЕКУЛЯРНАЯ МЕХАНИКА)}

\footnotetext{
О. Е. Глухова ${ }^{1}$, А. С. Колесникова ${ }^{2}$, М. М. Слепченков ${ }^{3}$, Г. В. Савостьянов ${ }^{4}$

${ }^{1}$ Доктор фризико-математических наук, заведующий кафредры радиотехники и электродинамики, Саратовский государственный университет им. Н. Г. Чернышевского, oeglukhova@yandex.ru

2 Программист отдела математического моделирования, Саратовский государственный университет им. Н. Г. Чернышевского), kolesnikova.88@mail.ru

${ }^{3}$ Ассистент касредры радиотехники и электродинамики, Саратовский государственный университет им. Н. Г. Чернышевского), slepchenkovm@mail.ru

${ }^{4}$ Аспирант касредры радиотехники и электродинамики, Саратовский государственный университет им. Н. Г. Чернышевского), follow.a.white.rabbbitt@gmail.com

В рамках гибридного метода(QM/MM) разработана новая модель, определяющая активную область структуры, т. е. ту область, для описания которой нужно применить высокоточные квантовые методы. В основе модели лежит решение задачи определения атомов с критическими значениями напряжения. Потенциальная энергия этих атомов и их ближайшего окружения рассчитывалась квантово-химическим методом, а потенциальная энергия оставшейся части структуры молекулярно-механическим методом. Гибридный метод (QM/MM) позволяет выявить с высокой точностью оптимальную топологию структуры и увеличить скорость нахождения ее равновесного состояния, а также исследовать динамику поведения десрормированной структуры во времени.
}

Ключевые слова: квантово-химические методы, молекулярно-механические методы, поле локальных напряжений, силы, углеродные наноструктуры. 


\section{1. АНАЛИТИЧЕСКИЙ ОБЗОР ГИБРИДНЫХ МЕТОДОВ QМ/ММ}

В настоящее время известны четыре класса методов расчета полной энергии наноструктур: методы ab initio (первопринципные методы) [1,2], методы функционала плотности [3], полуэмпирические [4-7] и эмпирические методы [8-11]. Последнее десятилетие развивается еще один класс методов, называемый классом гибридных методов (QM/MM), сочетающих молекулярно-механический и квантово-химический методы [12-15]. Преимущество гибридного метода по сравнению с методами ab initio (первопринципные методы), функционала плотности и полуэмпирического заключается в том, что с помощью него можно исследовать структуру, состоящую из большего количества атомов. В отличие от эмпирического метода гибридный метод позволяет более точно учитывать изменения в атомном каркасе наноструктур.

Большинство гибридных методов основаны на разбиении исследуемых систем на три локализованные области (активная, буферная области и окружающая область) и отличаются способом расчета потенциальной энергии. Активной областью наноструктуры называется область, в которой наблюдается изменение структуры нанокластера, например, образование дефекта, допирование или адсорбция атомов и т. д. Потенциальная энергия, описывающая химическое взаимодействие между атомами, которые располагаются в активной области, рассчитывается квантово-химическим методом. Вокруг активной области имеется буферная область с расстоянием от края активной области до края буферной области порядка 0.02 нм $[12,13]$. Потенциальная энергия буферной области рассчитывается комбинацией квантово-химического и молекулярно-механического методов. Потенциальная энергия оставшейся области, которая называется окружающей областью, находится молекулярно-механическим методом.

Rode и его коллеги $[12,13]$ рассчитали потенциальную энергию молекулярной системы гибридным методом, в котором используются молекулярно-механический метод (MM) и квантово-химический метод (QM), по формуле

$$
V=V^{\text {entire }}(M M)+\left(V^{A+B}(Q M)-V^{A+B}(M M)\right),
$$

где $V^{\text {entire }}(M M)$ - потенциальная энергия всей системы, рассчитанная при помощи $M M$ метода, $V^{A+B}(Q M)$ - потенциальная энергия молекулярной системы, состоящей из атомов, расположенных в активной и буферной областях, рассчитанная при помощи $\mathrm{QM}$ метода, $V^{A+B}(M M)$ - потенциальная энергия молекулярной системы, составленной из атомов, попавших в активную и буферную области, рассчитанная при помощи молекулярно-механического метода. Для выбранных потенциалов нулевой уровень энергии выбирается так, чтобы энергия молекулярной системы в равновесном состоянии для обоих методов равнялась нулю.

Kerdcharoen и Morokume's $[14,15]$ предложили находить потенциальную энергию молекулярной системы гибридным методом QM/MM по формуле

$$
V=P V^{A+B}+(1-P) V^{A},
$$

где $V^{A}$ - потенциальная энергия молекулярной системы, состоящей из атомов, расположенных в активной области, рассчитанной при помощи потенциала $\mathrm{QM}, V^{A+B}$ - потенциальная энергия молекулярной системы, состоящей из атомов, расположенных в активной и буферной областях, рассчитанной при помощи потенциала $\mathrm{QM}, P$ - арифметическое среднее сглаживающих функций $P_{i}\left(\alpha_{i}\right)$.

Heyden и Lin [16] записывают потенциальную энергию системы в следующем виде:

$$
\begin{gathered}
V=V^{A}+\sum_{i=1, \ldots, N} P_{i}\left(V_{i}^{A}-V^{A}\right)+\sum_{i=1, \ldots, N-1 ; j=i+1, \ldots, N} P_{i} P_{j}\left(V_{i, j}^{A}-\left[V^{A}+\sum_{r=i, j}\left(V_{r}^{A}-V^{A}\right)\right]\right)+ \\
+\sum_{(p, q)=(i, j),(i, k),(j, k)}\left(V_{p, q}^{A}-\left[V^{A}+\sum_{r=i, j}\left(V_{r}^{A}-V^{A}\right)\right]\right)+\ldots,
\end{gathered}
$$

где $V^{A}$ - потенциальная энергия молекулярной системы, состоящей из атомов, расположенных в активной области, рассчитанной при помощи потенциала $\mathrm{QM}, V_{i}^{A}-$ потенциальная энергия молекулярной системы, состоящей из атомов, расположенных в активной области, и одного атома $i$ из 
буферной области, рассчитанной при помощи потенциала $\mathrm{Q} M, V_{i, j}^{A}-$ потенциальная энергия молекулярной системы, состоящей из атомов, расположенных в активной области, и двух атомов $i$ и $j$ из буферной области, рассчитанной при помощи потенциала $\mathrm{Q} M, P_{i}-$ сглаживающая функция $i$-го атома.

При исследовании с помощью гибридных методов различных свойств наноструктур остается нерешенной проблема корректного с физической точки зрения обоснования местоположения активной области и динамики её изменения с течением времени. В связи с этим целью данной работы является разработка концепции определения зависимости геометрии активной области от геометрии наноструктуры. Потенциальная энергия активной области рассчитывается квантово-химическими методами в рамках метода $\mathrm{QM} / \mathrm{MM}$.

Метод QM/MM может быть реализован в рамках молекулярной динамики, что позволит осуществить численный эксперимент с многоатомными структурами в режиме реального времени с учетом внешних условий: постоянных и переменных электрических полей, теплового воздействия, постоянной и изменяющиеся механической нагрузки, плазма, адсорбирующиеся атомы.

\section{2. КОНЦЕПЦИЯ РАСЧЕТА МОЛЕКУЛЯРНОЙ ДИНАМИКИ ГИБРИДНЫМ МЕТОДОМ (QМ/ММ) НА ОСНОВЕ АНАЛИЗА ПОЛЯ ЛОКАЛЬНЫХ НАПРЯЖЕНИЙ}

Рассмотрим основные особенности реализации молекулярной динамики гибридным методом (QM/MM) на основе анализа поля локальных напряжений. В качестве квантово-химического метода используется метод сильной связи, представленный в работе [17], в качестве молекулярномеханического метода используется метод REBO [18].

Каждый шаг молекулярно-динамического расчета гибридным методом основывается на анализе поля локальных напряжений. Потенциальная энергия гибридным методом находится по формуле (1). Для текущего состояния атомного каркаса рассчитывается поле локальных напряжений с помощью оригинальной методики [19], с целью выявления наиболее деформированных и/или дефектных участков атомной сетки.

Методика расчета поля локальных напряжений основана на эмпирическом подходе в расчете энергии одного атома [19]. Расчет поля локальных напряжений для некоторого неравновесного состояния наноструктуры осуществлялся по следующему алгоритму.

1. Оптимизация исходной атомной структуры.

2. Вычисление распределения объемной плотности энергии по атомам.

3. Вычисление распределения объемной плотности энергии по атомам структуры, подвергнутой внешнему воздействию.

4. Расчет поля локальных напряжений атомного каркаса по разности значений объемных плотностей энергии атомов структуры, подвергнутой внешнему воздействию, и структуры в равновесном состоянии.

В результате сканирования карты локальных напряжений выделяются атомы, которые испытывают критические напряжения, такие атомы называются «горячими» атомами. Относительно каждого из этих атомов строится сфера радиусом $r_{i n}$, который, например, для углерода может быть выбран равным 0.2 нм, поскольку для углерода при увеличении длины связи до 0.2 нм происходит её разрыв. Радиус буферной зоны $r_{\text {out }}$ выбирается на 0.02 нм больше $[12,13]$ радиуса активной области. В общем случае имеем несколько активных областей с соответствующими им буферными областями, поскольку «горячие» атомы могут образовываться в разных участках структуры.

В процессе молекулярно-динамического расчета одним из основных моментов является расчет сил, действующих на атомы молекулярной системы. При использовании потенциала (1) сила, действующая на выделенный атом, рассчитывается по формуле

$$
f_{i}=f_{i}^{\text {entire }}(M M)+S\left(r_{i}\right)\left(f_{i}^{A+B}(Q M)-f_{i}^{A+B}(M M)\right),
$$

где $f_{i}^{\text {entire }}(M M)$ - сила, действующая на атом со стороны всей структуры и определяющаяся потенциалом Бреннера [18], $f_{i}^{A+B}(Q M)-$ сила, действующая на атом со стороны активной и буферной областей, рассчитанная с помощью квантово-химического метода сильной связи [17], $f_{i}^{A+B}(M M)-$ 
сила, действующая на атом со стороны в активной и буферной области, рассчитанная при помощи потенциала Бреннера, $S\left(r_{i}\right)$ - масштабирующая функция, которая находится по формуле

$$
S\left(r_{i}\right)= \begin{cases}1, & r_{i} \leq r_{\text {in }} \\ \frac{\left(r_{\text {out }}^{2}-r_{i}^{2}\right)^{2}\left(r_{\text {out }}^{2}-2 r_{i}^{2}-3 r_{\text {in }}^{2}\right)}{\left(r_{\text {out }}^{2}-r_{\text {in }}^{2}\right)^{3}}, & r_{\text {in }}<r<r_{\text {out }} \\ 0, & r_{i} \geq r_{\text {out }},\end{cases}
$$

где $r_{\text {in }}$ и $r_{\text {out }}-$ внутренний и внешний радиусы буферной области, а $r_{i}-$ расстояние от ближайшего «горячего» атома до $i$-го атома.

В общем случае при заданной потенциальной функции силы, действующие на каждый атом, рассчитываются через градиент потенциальной энергии:

$$
f_{i}=-\frac{\partial U}{\partial r_{i}}
$$

где $r_{i}-$ координаты $i$-го атома.

Расчет градиента потенциальной энергии для потенциала REBO не составляет особой сложности. Остановимся подробно на расчете градиента потенциальной энергии в рамках квантово-химического метода сильной связи.

Энергия в рамках метода сильной связи [17] определяется соотношением

$$
E_{t o t}=E_{b o n d}+E_{r e p}
$$

где $E_{b o n d}-$ энергия занятых электронных состояний, $E_{r e p}-$ энергия отталкивания, которая является результатом электронного взаимодействия между атомами.

Энергия отталкивания определяется формулой

$$
E_{r e p}=\sum_{i<j} V_{r e p}\left(\left|r_{i}-r_{j}\right|\right),
$$

где

$$
V_{\text {rep }}(r)=p_{5}\left(\frac{p_{3}}{r}\right)^{p_{6}} \exp \left\{p_{6}\left[\left(\frac{p_{3}}{p_{2}}\right)^{p_{4}}-\left(\frac{r}{p_{2}}\right)^{p_{4}}\right]\right\} .
$$

Энергия занятых электронных состояний определяется по формуле

$$
E_{\text {bond }}=2 \sum_{m} \varepsilon_{n}
$$

где $m$ - число занятых орбиталей, $\varepsilon_{n}-$ энергии молекулярных орбиталей, которые соответствуют собственным значениям матрицы гамильтониана системы [17]. Элементы матрицы гамильтониана определяются формулой

$$
V_{i j \alpha}(r)=V_{i j \alpha}^{0}\left(\frac{p_{3}}{r}\right)^{p_{1}} \exp \left\{p_{1}\left[\left(\frac{p_{3}}{p_{2}}\right)^{p_{4}}-\left(\frac{r}{p_{2}}\right)^{p_{4}}\right]\right\},
$$

где $\alpha$ - тип перекрывания атомных орбиталей, значения параметров $V_{i j \alpha}^{0}, p_{1}, \ldots, p_{6}$ приведены в [17].

Остановимся подробно на процедуре нахождения градиента энергии $E_{\text {bond }}$ :

$$
\frac{\partial E_{\text {bond }}}{\partial r_{i}}=2 \sum_{m} \frac{\partial \varepsilon_{n}}{\partial r_{i}} .
$$

Для определения $\partial \varepsilon_{n} / \partial r_{i}$ необходимо определить зависимость между изменением собственных значений и изменением элементов матрицы гамильтониана.

В разработанной Дж. Х. Уилкинсоном матричной теории возмущений [20], возмущение матрицы $A$, зависящее от параметра малости возмущений $\delta$, представляется в виде

$$
A(\delta)=A+A_{1} \delta
$$

где $A_{1}-$ некоторая матрица. 
В рамках алгебраического подхода Уилкинсон получил выражение для возмущенных собственных значений в виде сходящегося в некоторой окрестности ряда:

$$
\lambda_{i}(\delta)=\lambda_{i}+k_{1}^{i} \delta+k_{2}^{i} \delta^{2}+\ldots
$$

где $\lambda_{i}$ - собственное значение матрицы $A, \lambda_{i}(\delta)-$ собственное значение, соответствующее возмущенной матрице $A(\delta)$. Здесь $k_{1}^{i}, k_{2}^{i}, \ldots-$ коэффициенты, определяющиеся соотношением

$$
k_{j}^{i}=\frac{1}{s_{i}} \sum_{\substack{i=1 \\ i \neq j}}^{m} t_{j m-1}^{i} \beta_{j i}
$$

где

$$
\begin{gathered}
t_{j m}^{i}=\frac{t_{j m-1}^{i}\left(k_{1}^{i}-\beta_{j j}\right)+\ldots+t_{j 1}^{i} k_{m-1}^{i}}{\left(\lambda_{j}-\lambda_{i}\right) s_{j}}, \\
s_{i}=y_{i}^{T} x_{i}, \\
\beta_{i j}=y_{i}^{T} A_{1} x_{j},
\end{gathered}
$$

где $y_{i}, x_{i}$ - соответственно левые и правые собственные векторы.

Пусть $H(r)$ - матрица гамильтониана для некоторого фиксированного состояния молекулярной системы, где $r=\left[r_{1}, r_{2}, \ldots, r_{N}\right]$, здесь $N$ - число атомов системы, $r_{i}-$ радиус-вектор $i$-го атома. Обозначим через $e_{i l}$ аналогичный $r$ набор векторов, у которого компоненты всех векторов равны нулю, за исключением $l$-й компоненты $i$-го вектора, равной единице.

Будем рассматривать возмущение матрицы гамильтониана $H(r)$, соответствующее сдвигу $l$-й компоненты $i$-го радиус-вектора системы на величину $\delta$, в виде

$$
H_{\delta}^{i l}(r) \delta=H\left(r+e_{i l} \delta\right)-H(r) .
$$

Элементы матрицы гамильтониана имеют обобщенный вид

$$
H_{p q}(r)=h_{p q}\left(r_{1}, \ldots, r_{N}\right) \text {, }
$$

где $h_{p q}\left(r_{1}, \ldots, r_{N}\right)$ - дифференцируемы. Тогда элементы матрицы $H_{\delta}^{i l}(r)$ можно записать в виде

$$
H_{p q \delta}^{i l}(r)=\frac{h_{p q}\left(r_{1}, \ldots, r_{i}+e_{l} \delta, \ldots, r_{m}\right)-h_{p q}\left(r_{1}, \ldots, r_{m}\right)}{\delta}
$$

где $e_{l}-$ вектор, направленый вдоль $l$-й оси декартовой системы координат. Используя разложение в ряд Тейлора, получаем:

$$
H_{p q \delta}^{i l}(r)=\left(\frac{\partial h_{p q}\left(r_{1}, \ldots, r_{m}\right)}{\partial r_{i l}} \delta+O\left(\delta^{2}\right)\right) / \delta .
$$

При делении на $\delta$ окончательно получаем в матричном виде:

$$
H_{\delta}^{i l}(r)=\frac{\partial H(r)}{\partial r_{i l}}+E O(\delta)
$$

где $\partial H(r) / \partial r_{i l}$ - поэлементное частное дифференцирование матрицы $H(r)$ по $l$-й координате радиусвектора $i$-го атома, а $E-$ единичная матрица.

Применяя формулу (3), получаем:

$$
\varepsilon_{n}\left(r+e_{i l} \delta\right)=\varepsilon_{n}(r)+k_{1}^{n}\left(H_{\delta}^{i l}(r)\right) \delta+k_{2}^{n}\left(H_{\delta}^{i l}(r)\right) \delta^{2}+\ldots
$$

или

$$
\varepsilon_{n}\left(r+e_{i l} \delta\right)-\varepsilon_{n}(r)=k_{1}^{n}\left(H_{\delta}^{i l}(r)\right) \delta+k_{2}^{n}\left(H_{\delta}^{i l}(r)\right) \delta^{2}+\ldots
$$

Разделим выражение (8) на $\delta$ и устремим $\delta$ к нулю

$$
\lim _{\delta \rightarrow 0} \frac{\varepsilon_{n}\left(r+e_{i l} \delta\right)-\varepsilon_{n}(r)}{\delta}=\lim _{\delta \rightarrow 0}\left\{k_{1}^{n}\left(H_{\delta}^{i l}(r)\right)+k_{2}^{n}\left(H_{\delta}^{i l}(r)\right) \delta+\ldots\right\} .
$$


Поскольку $k_{i}^{n}-$ коэффициенты аналитически-сходящегося ряда, то они ограничены. Таким образом, имеем:

$$
\frac{\partial \varepsilon_{n}(r)}{\partial r_{i l}}=\lim _{\delta \rightarrow 0} k_{1}^{n}\left(H_{\delta}^{i l}(r)\right)
$$

Из формулы (4) получаем:

$$
k_{1}^{n}\left(H_{\delta}^{i l}(r)\right)=\frac{\beta_{n n}\left(H_{\delta}^{i l}(r)\right)}{s_{n}} .
$$

Поскольку матрица гамильтониана является симметричной, то левый и правый собственные векторы совпадают и имеют норму, равную 1. Поэтому из формулы (5) получаем, что $s_{n}=1$.

Итого

откуда

$$
k_{1}^{n}\left(H_{\delta}^{i l}(r)\right)=\beta_{n n}=x_{n}^{T}\left(\frac{\partial H(r)}{\partial r_{i l}}+E O(\delta)\right) x_{n},
$$

$$
\frac{\partial \varepsilon_{n}(r)}{\partial r_{i l}}=\lim _{\delta \rightarrow 0}\left\{x_{n}^{T} \frac{\partial H(r)}{\partial r_{i l}} x_{n}+x_{n}^{T} E x_{n} O(\delta)\right\},
$$

так как $x_{n}^{T} E x_{n}=x_{n}^{T} x_{n}=s_{n}=1$, получаем:

$$
\frac{\partial \varepsilon_{n}(r)}{\partial r_{i l}}=\lim _{\delta \rightarrow 0}\left\{x_{n}^{T} \frac{\partial H(r)}{\partial r_{i l}} x_{n}+O(\delta)\right\}
$$

Первое выражение под пределом не зависит от $\delta$, а второе стремится к нулю при $\delta \rightarrow 0$, поэтому

$$
\frac{\partial \varepsilon_{n}(r)}{\partial r_{i l}}=x_{n}^{T} \frac{\partial H(r)}{\partial r_{i l}} x_{n}
$$

Таким образом, для нахождения градиента $E_{\text {bond }}$ достаточно знать собственные векторы и поэлементные частные производные гамильтониана в точке $r$.

Несмотря на квадратичную асимптотику формулы (9), расчет производной для собственных значений по этой формуле в методе сильной связи весьма эффективен в силу большой разреженности матрицы гамильтониана.

\section{3. ВЫводы}

В данной работе впервые изложена концепция динамического определения активной области в рамках гибридного метода $(\mathrm{QM} / \mathrm{MM})$, основанная на расчете поля локальных напряжений. Сканирование карты локальных напряжений позволяет динамически определять активные, буферные и окружающие области, в то время как в существующих моделях [12-16] эти области фиксированы. В связи с этим представленная в данной работе модель позволяет во времени определять изменения или перестроения связей атомного каркаса структуры, например, в области деформации структуры. Следует отметить, что описанный алгоритм определения активных областей применим и для моделей, представленных в работах [14-16]. Изложены основные особенности реализации молекулярной динамики гибридным методом, с примением квантово-химического метода сильной связи. Разработанная гибридная молекулярно-динамическая модель $M M / Q M$ обеспечивает симулирование работы наноустройств, изучение свойств материалов.

Работа выполнена при финансовой поддержке РФФИ (проект 12-01-31036, 12-02-00807, 13 08-00986) и Федеральной целевой программы «Научные и научно-педагогические кадры инновацзионной России на 2009-2013 годь», XLI очередь мероприятие 1.2.1, технические науки, номер соглашения «14.B37.21.1094», Президентской стипендии 2013-2016 (проект СП-2302.2013.1).

\section{Библиографический список}

1. Хурсан С. Л. Квантовая механика и квантовая химия. Конспекты лекций. Уфа : ЧП Раянов, 2005. 164 с. 2. Аминова P. М. Основы современной квантовой химии / Казан. гос. ун-т. Казань, 2004. 106 с.

3. Сатанин A. М. Введение в теорию функционала плотности : учеб.-метод. пособие / Нижегород. гос. ун-т им. Н. И. Лобачевского. Н. Новгород, 2009. 64 с. 4. Блатов В. А., Шевченко А. П., Пересыпкина Е. В. Полуэмпирические расчетные методы квантовой химии : учеб. пособие. Самара : Универс-групп, 2005. $32 \mathrm{c}$.

5. Глухова О. Е., Жбанов А. И. Равновесное состоя- 
ние нанокластеров $C_{60}, C_{70}, C_{72}$ и локальные дефекты молекулярного остова // Физика твердого тела. 2003. Т. 45, вып. 1. С. 189-196.

6. Goodwin L. A. New tight-binding parametrization for carbon // J. Phys. : Condens. Matter. 1991. Vol. 3. P. 3869-3878.

7. Харрисон У. Электронная структура и свойства твердых тел. М. : Мир, 1983. 381 с.

8. Tersoff $J$. Modeling solid-state chemistry : Interatomic potentials for mnlticomponent systems // Phys. Rev. B. 1989. Vol. 39, № 8. P. 5566-5568.

9. Brenner $D$. $W$. Empirical potential for hydrocarbons for use in simulating the chemical vapor deposition of diamond films // Phys. Rev. B. 1990. Vol. 42, № 15. P. 9458-9471.

10. Stuart S. J., Tutein A. B., Harrison J. A. A reactive potential for hydrocarbons with intermolecular interactions // J. Chem. Phys. 2000. Vol. 112, № 14. P. 6472-6486.

11. Глухова О. Е. Изучение механических свойств углеродных нанотрубок стручкового типа на молекулярномеханической модели // Физика волновых процессов и PC. 2009. T. 12, № 1. C. 69-75.

12. Kerdcharoen T., Liedl K. R., Rode B. M. A QM/MM simulation method applied to the solution of $\mathrm{Li}^{*}$ in liquid ammonia // Chem. Phys. 1996. Vol. 211. P. 313-323.

13. Hofer T. S., Pribil A. B., Randolf B. R., Rode B. M. Structure and Dynamics of Solvated $\mathrm{Sn}(\mathrm{II})$ in Aqueous Solution : An ab Initio QM/MM MD Approach // J. Am. Chem. Soc. 2005. Vol. 127. P.14231-14238.
14. Kerdcharoen T., Morokuma K. J. Combined QM/MM Simulation of $\mathrm{Ca}^{2+} /$ Ammonia Solution based on ONIOM-XS Method : Octahedral Coordination and Implication to Biology // Chem. Phys. 2003. Vol. 118. P. 8856-8863.

15. Kerdcharoen T., Morokuma K. ONIOM-XS : an extension of the ONIOM method for molecular simulation in condensed phase // Chem. Phys. Lett. 2002, Vol. 355. P. 257-262.

16. Heyden A., Lin H., Truhlar D. G.Adaptive partitioning in combined quantum mechanical and multiscale simulations // J. Phys. Chem. B. 2007. Vol. 111. P. 2231-2241.

17. Glukhova O. E., Kolesnikova A. S., Kossovich E. L., Zhnichkov $R$. $Y$. Super strong nanoindentors for biomedical applications based on bamboo-like nanotubes// Proc. of SPIE. 2012. Vol. 8233. P. 823311(8).

18. Stuarta S. J., Tutein A. B., Harrison J. A. A reactive potential for hydrocarbons with intermolecular interactions // J. Chem. Phys. 2000. Vol. 112, № 14. P. 6472-6486

19. Glukhova O. E., Slepchenkov M. M. Influence of the curvature of deformed graphene nanoribbons on their electronic and adsorptive properties : theoretical investigation based on the analysis of the local stress field for an atomic grid // Nanoscale. 2012. Vol. 11. P. 33353344 .

20. Уилкинсон Дж. Х. Алгебраическая проблема собственных значений. М. : Наука, Физматлит, 1970. $564 \mathrm{c}$.

\section{Technique of Definition Areas Requiring a Quantum Description Within of the Hybrid Method (Quantum Mechanics / Molecular Mechanics)}

\section{O. E. Glukhova, A. S. Kolesnikova, M. M. Slepchenkov, G. V. Savostianov}

Saratov State University, Russia, 410012, Saratov, Astrahanskaya st., 83, oeglukhova@yandex.ru, kolesnikova.88@mail.ru, slepchenkovm@mail.ru, follow.a.white.rabbbitt@gmail.com

The new model, which determines the active area (the region for which high-precision quantum methods must be used) of the structure,was developed within the of the hybrid method (QM/MM). Problem of determining atoms with the critical tension values is the basis of this model. The potential energy of these atoms and its nearest neighbours was calculated by quantum-chemical method. The potential energy of the rest structure was calculated by molecular mechanical method. The Hybrid method (QM/MM) allows to reveal with high accuracy optimum topology structure and increase the speed of finding its equilibrium state.

Key words: the quantum-chemical methods, the molecular mechanics methods, the critical tension values, the force, the carbon nanostructures.

\section{References}

1. Khursan S. L. Kvantovaj mekhanika i kvantovaj khimij [Quantum mechanics and quantum chemistry. Lecture notes]. Ufa, PE, Rayanov, 2005, 164 p. (in Russian).

2. Aminova R. M. Osnovy sovremennoj kvantovoj khimii [The foundations of modern quantum chemistry]. Kazan, Kazan University Press, 2004, 106 p. (in Russian).

3. Satanin A. M. Vvedenie v teoriyu funktinala plotnosti. Uchebno-metodicheskoe posobie. [Introduction to density functional theory. Teaching manual]. Nizhny
Novgorod, Nizhny Novgorod State University named after N. I. Lobachevsky, 2009, 64 p. (in Russian). 4. Blatov V. A., Shevchenko A. P., Peresypkina E. V. Poluempiricheskie raschetnye metody kvantovoj ximii. Uchebnoe posobie [Semi-empirical calculation methods of quantum chemistry. Teaching manual]. Samara, Universal Group, 2005, 32 p. (in Russian).

5. Glukhova O. E., Zhbanov A. I. The equilibrium state of nanoclusters $C_{60}, C_{70}, C_{72}$ and local defects of the 
molecular skeleton. Physics of the Solid State [Fizika tverdogo tela], 2003, vol. 45, iss. 1, pp. 189-196.

6. Goodwin L. A. New tight-binding parametrization for carbon. J. Phys. : Condens. Matter., 1991, vol. 3, pp. 3869-3878.

7. Harrison U.Elektronnaia struktura $i$ svojstva tverdyx tel. [Electronic structure and properties of solids]. Moscow, Mir, 1983, 381 p. (in Russian).

8. Tersoff J. Modeling solid-state chemistry : Interatomic potentials for multicomponent systems. Phys. Rev. B., 1989, vol. 39, no. 8, pp. 5566-5568.

9. Brenner D. W. Empirical potential for hydrocarbons for use in simulating the chemical vapor deposition of diamond films. Phys. Rev. B., 1990, vol. 42, no. 15, pp. 9458-9471.

10. Stuart S. J., Tutein A. B., Harrison J. A. A reactive potential for hydrocarbons with intermolecular interactions. J. Chem. Phys., 2000, vol. 112, no. 14, pp. 6472-6486.

11.Glukhova O. E. The study of mechanical properties of carbon nanotubes cayenne-type molecular-mechanical model. Physics of wave processes and PC., 2009, vol. 12, iss. 1, pp. 69-75.(in Russian).

12. Kerdcharoen T., Liedl K. R., Rode B. M. A QM/MM simulation method applied to the solution of $\mathrm{Li}^{*}$ in liquid ammonia. Chem. Phys., 1996, vol. 211, pp. 313-323.

13. Hofer T. S., Pribil A. B., Randolf B. R., Rode B. M. Structure and Dynamics of Solvated $\mathrm{Sn}(\mathrm{II})$ in Aqueous Solution : An ab Initio QM/MM MD Approach. J. Am. Chem. Soc., 2005, vol. 127, pp. 14231-14238.

14.Kerdcharoen T., Morokuma K. J. Combined QM/MM
Simulation of $\mathrm{Ca}^{2+} /$ Ammonia Solution based on ONIOM-XS Method : Octahedral Coordination and Implication to Biology. Chem. Phys., 2003, vol. 118, pp. 8856-8863.

15.Kerdcharoen T., Morokuma K. ONIOM-XS : an extension of the ONIOM method for molecular simulation in condensed phase. Chem. Phys. Lett., 2002, vol. 355, pp. 257-262.

16.Heyden A., Lin H., Truhlar D. G. Adaptive partitioning in combined quantum mechanical and multiscale simulations. J. Phys. Chem. B., 2007, vol. 111, pp. 2231-2241.

17. Glukhova O. E., Kolesnikova A. S., Kossovich E. L., Zhnichkov R. Y. Super strong nanoindentors for biomedical applications based on bamboo-like nanotubes. Proc. of SPIE., 2012, vol. 8233, pp. 823311(8).

18. Stuarta S. J., Tutein A. B., Harrison J. A. A reactive potential for hydrocarbons with intermolecular interactions. Journal of chemical physics, 2000, vol. 112, no. 14. pp. 6472-6486

19. Glukhova O. E., Slepchenkov M. M. Influence of the curvature of deformed graphene nanoribbons on their electronic and adsorptive properties : theoretical investigation based on the analysis of the local stress field for an atomic grid. Nanoscale, 2012, vol. 11, pp. 33353344 .

20. Wilkinson J. X. Algebraicheskaj problema sobstvennyx znachenij [The algebraic eigenvalue problem]. Moscow, Nauka, FIZMATLIT, 1970, 564 p. (in Russian).

УДК 622.4:536.21

\title{
ОПРЕДЕЛЕНИЕ ИЗМЕНЕНИЯ ТЕМПЕРАТУРЫ СТЕНКИ ПОЛОСТИ В ТВЕРДОМ ТЕЛЕ ПРИ ИЗМЕНЕНИИ ТЕМПЕРАТУРЫ ДВИЖУЩЕГОСЯ В ПОЛОСТИ ГАЗА
}

\author{
О. И. Дударь ${ }^{1}$, Е. С. Дударь ${ }^{2}$, М. А. Осипенко ${ }^{3}$ \\ ${ }^{1}$ Кандидат фризико-математических наук, доцент кафедры теоретической механики, Пермский национальный исследова- \\ тельский политехнический университет, olegdudar@yandex.ru \\ ${ }^{2}$ Кандидат технических наук, доцент кафредры начертательной геометрии, Пермский национальный исследовательский \\ политехнический университет, elendudar@yandex.ru \\ ${ }^{3}$ Кандидат фризико-математических наук, доцент кафедры теоретической механики, Пермский национальный исследова- \\ тельский политехнический университет, ота@ theormech.pstu.ac.ru \\ Найдено изменение температуры стенки цилиндрической полости в твердом теле как отклик на изменение температуры про- \\ текающего в полости газа. Рассмотрены 3 важных частных случая изменения температуры газа со временем: температура \\ постоянна; температура изменяется по линейному закону; температура изменяется по гармоническому закону. Представ- \\ лены грасики пяти «ө-фрункций», через которые записываются решения. Грасики получены с помощью квадратурной \\ фрормулы Гаусса численным интегрированием несобственных интегралов, содержащих цилиндрические фуннции.
}

Ключевые слова: цилиндрическая полость, нестационарная теплопроводность, конвективный теплообмен, фрункции Бесселя и Неймана, несобственные интегралы. 